\title{
An Approach to New Methods for Digital Signal Processing on Optical Mapping Sequences and Electrical Mapping
}

\author{
Sergio Muñoz-Romero ${ }^{1}$, Margarita Sanromán-Junquera ${ }^{1}$, Cristina Soguero-Ruiz $^{1}$, \\ Inmaculada Mora-Jiménez ${ }^{1}$, Raúl Caulier-Cisterna ${ }^{1}$, Javier Moreno-Planas ${ }^{2}$, \\ Jorge García-Quintanilla ${ }^{3}$, Arcadi García-Alberola ${ }^{4}$, José Luis Rojo-Álvarez ${ }^{1}$ \\ ${ }^{1}$ Universidad Rey Juan Carlos, Madrid, Spain \\ ${ }^{2}$ Hospital Ramón y Cajal, Madrid, Spain \\ ${ }^{3}$ Hospital Clínico San Carlos, Madrid, Spain \\ ${ }^{4}$ Hospital Clínico Universitario Virgen de la Arrixaca, Murcia, Spain
}

\begin{abstract}
Optical Mapping (OM) sequences represent a very useful tool for registering cardiac bioelectric activity with high patio-temporal resolution. Conventional preprocessing of OM movies is mostly done with spatio-temporal filters, which could be improved for yielding better signal to noise ratio in the processed images. Also, the multimodal mapping, such as electrical mapping combined with OM, is limited by the need of presence of catheters in the optical image. In this work, we addressed a twofold objective. First, we explored the suitability and scope of intrinsic multirecording techniques to separate different noise sources in OM sequences. Second, we explored the use of advanced techniques to extrapolate the electrical mapping shadowed spatial regions on the OM images. We scrutinized the Singular Value Decomposition (SVD) on OM sequences obtained in OM laboratory, showing that: (1) the second spatial component mostly conveys movement artifacts, and could be removed or processed for improving the quality; (2) the components from 3 to about 10 convey both spatial variations and spatial artifacts, which can not be readily decoupled. The use of GPatt, an advanced algorithm for multidimensional pattern extrapolation based on Gaussian Processes, was also analyze in a multimodal recording of OM and electrical mapping including a lasso catheter, showing interesting capabilities for removing the catheter shadows with fidelity, and dependence with respect to noise and texture. Our results show that other processing methods can provide with enhanced signal to noise ratio when working with OM sequences.
\end{abstract}

\section{Introduction}

Optical Mapping (OM) sequences are the basis for a set of experimental research techniques that are used in elec- trophysiology for analyzing the electrical behavior of the heart, either in normal or in pathological situations. OM consists of recording (via CCD or CMOS cameras) the fluorescence as emitted by photosensitive contrasts adhered to the cardiac membrane cells. A high-resolution camera with high sensitivity and fast time-acquisition rate yields a set of frames, such that the spatial dimension as represented by each pixel integrates the effect of transmembrane potential on the electric field [1,2].

Conventional processing methods have been mostly focused on preprocessing by using linear-based operators, such as spatio-temporal averaging kernels, or its robust versions such as median filtering. However, the presence of optical artifacts, nonlinear distortion, or presence of different noises with diverse natures, makes OM preprocessing an open research field for signal and image processing. In addition, the multimodal mapping is increasingly being used, such as electrical mapping combined with $\mathrm{OM}$, but it becomes limited by the need of presence of catheters in the optical image, which are not easy to remove [2, 3].

Therefore, we addressed here a twofold objective. First, we explored the suitability and scope of intrinsic multirecording techniques to separate different noise sources in OM sequences, specifically, in terms of the Singular Value
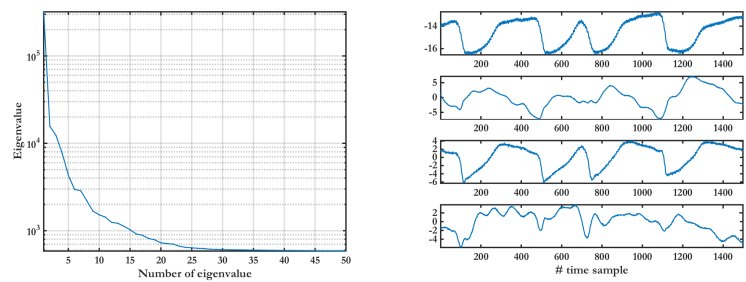

Figure 1. Eigenvalues and first 4 temporal eigenvectors of the non-filtered $\mathrm{OM}$ sequence. 

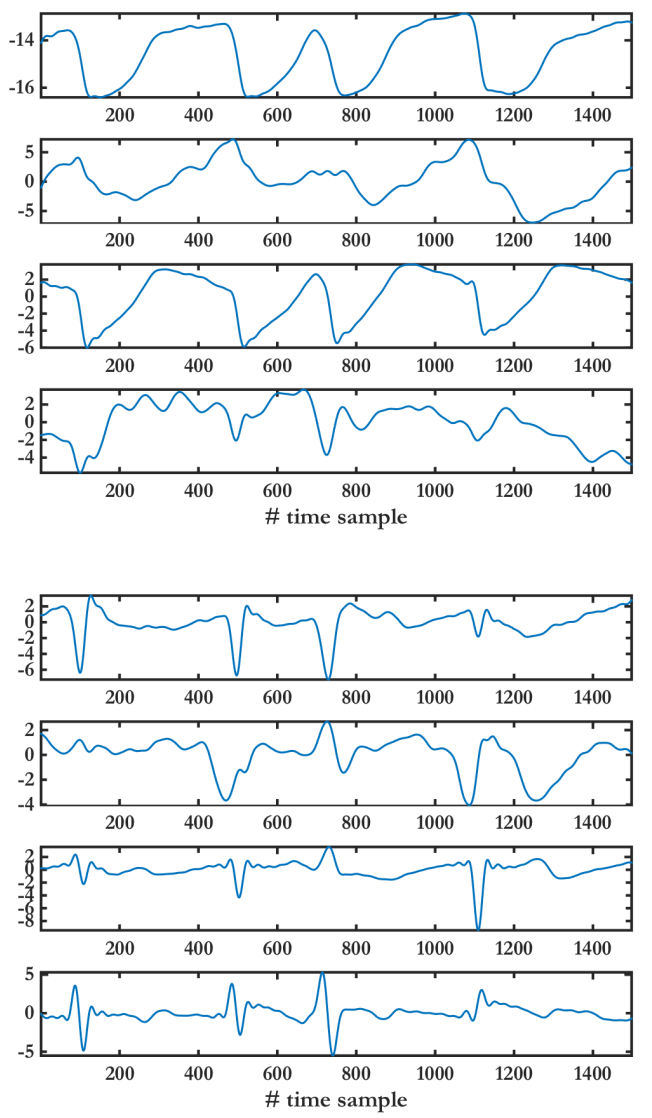
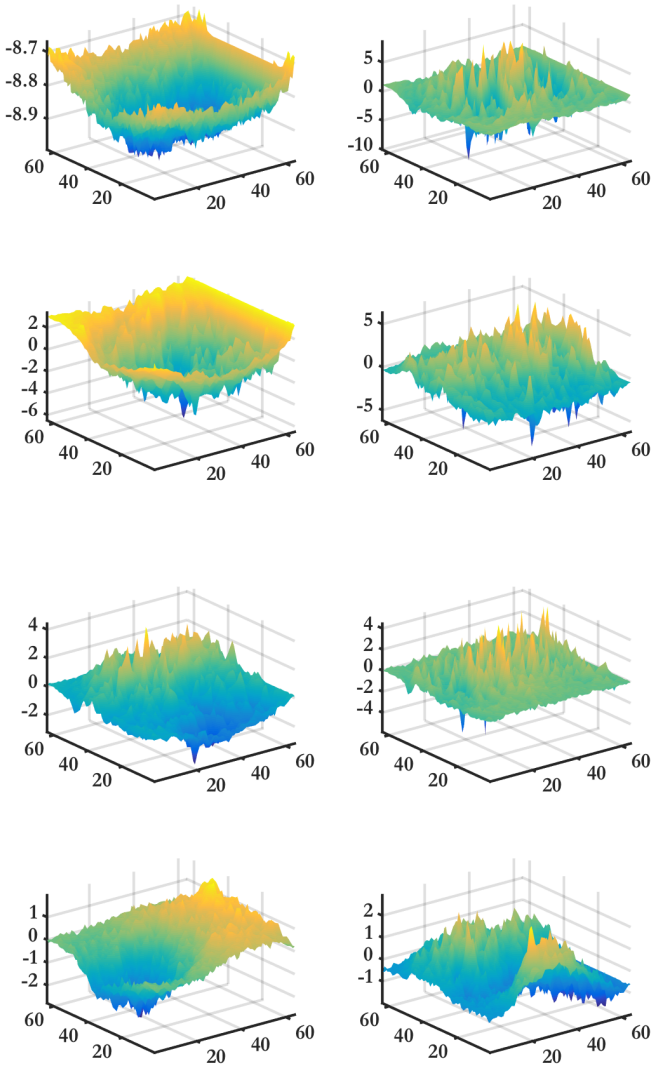

Figure 2. Temporal (left) and spatial (right, as a function of pixel indices) eigenvectors, from 1 to 8.

Decomposition (SVD) on OM sequences. Second, we explored the use of advanced techniques to extrapolate the electrical mapping shadowed spatial regions on the OM images, by using for this purpose GPatt, an advanced algorithm for multidimensional pattern extrapolation based on Gaussian Processes machine learning [4], which was analyzed in a multimodal recording of OM and electrical mapping including a lasso catheter.

The scheme of the paper is as follows. In Section 2, the properties of the SVD are scrutinized in a typical OM sequence, and spatial and temporal components are studied. In Section 3, the GPatt algorithm is used to cancel and to extrapolate the lasso catheter in a multimodal $\mathrm{OM}$ and electrical mapping sequence. Finally, in Section 4, conclusions and future work are sketched.

\section{Noise Cancellation}

One of the most useful matrix factorization which is often used in real practice is the SVD [5], given by

$$
\mathbf{X}=\mathbf{U} \boldsymbol{\Sigma} \mathbf{V}^{\top}
$$

where the columns of $\mathbf{U} \in \mathbb{R}^{n \times n}$ are known as the right eigenvectors of $\mathbf{X} \in \mathbb{R}^{n \times N}, \boldsymbol{\Sigma} \in \mathbb{R}^{n \times n}$ is a matrix with the singular values of $\mathbf{X}$, and $\mathbf{V} \in \mathbb{R}^{N \times n}$ has the left singular vectors of $\mathbf{X}$. In our case, $\mathbf{X}$ is a rectangular matrix obtained by reshaping in space the OM sequence, where $n$ corresponds to the aligned spatial dimensions of the image, and $N$ is the number of frames. Accordingly, the left (right) eigenvectors are the directions of spatial (temporal) distribution. An advantage of this technique is the possibility of working with non-square matrices, however, the left and right singular vectors are the eigenvectors of covariance matrix $\left(\mathbf{C}_{\mathbf{X X}}\right)$ and of internal products matrix $\left(\mathbf{K}_{x}\right)$, respectively:

$$
\begin{gathered}
\mathbf{C}_{\mathbf{X X}}=\mathbf{X} \mathbf{X}^{\top}=\mathbf{U} \boldsymbol{\Sigma} \mathbf{V}^{\top} \mathbf{V} \boldsymbol{\Sigma} \mathbf{U}^{\top}=\mathbf{U} \boldsymbol{\Sigma}^{2} \mathbf{U}^{\top}=\mathbf{U} \boldsymbol{\Lambda} \mathbf{U}^{\top} \\
\mathbf{K}_{x}=\mathbf{X}^{\top} \mathbf{X}=\mathbf{V} \boldsymbol{\Sigma} \mathbf{U}^{\top} \mathbf{U} \boldsymbol{\Sigma} \mathbf{V}^{\top}=\mathbf{V} \boldsymbol{\Sigma}^{2} \mathbf{V}^{\top}=\mathbf{V} \boldsymbol{\Lambda} \mathbf{V}^{\top} .
\end{gathered}
$$

Hence, it can be concluded that eigenvalues $\boldsymbol{\Lambda}=\boldsymbol{\Sigma}^{2}$ of $\mathbf{C}_{\mathbf{X X}}$ and $\mathbf{K}_{x}$ are the same, and SVD solves jointly and efficiently two problems of eigenvalue decomposition.

The SVD was applied to an OM sequence, sampled at 800 frames per second. Figure 1 shows the eigenvalues, indicating that most of the variance is in some few com- 

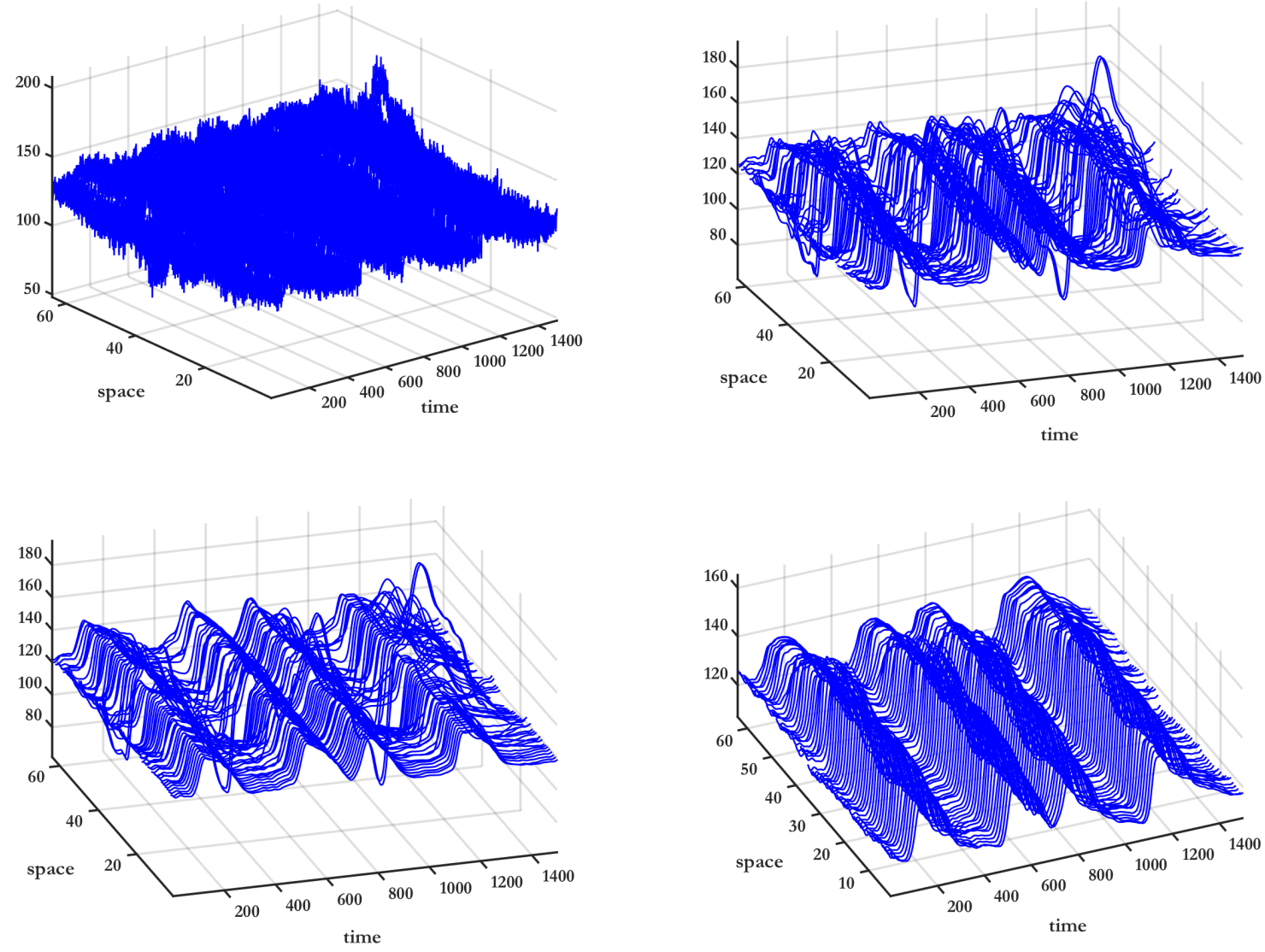

Figure 3. Signals along a line crossing the middle of the OM sequence for the inn-filtered sequence (a), and for the filtered sequence by considering the spatial eigenvectors from 1 to 5 (b), 1 and 2 (c), and 1 and 3 (d).

ponents, and an exponential decay of eigenvalues associated to details. The same figure shows the first 4 temporal eigenvectors when no filtering has been applied, hence it is evident that the high frequency noise is spread throughout many of the components. Therefore, the time signals were first filtered with a low-pass $30 \mathrm{~Hz}$ zero-phase filter.

Figure 2 shows the temporal and spatial eigenvectors, from 1 to 8 . It can be seen that in this case, components 1 and 3 convey the (smoothed) basic waveform of action potentials, whereas components 5, 7 and 8 have some energy peaks, corresponding to the transitions in the fast depolarization phase. Also, the first spatial directions include variance from the image distortion and spatial structure for the optical noise.

We further scrutinized in Fig. 3 the content of each spatial eigenvector. The first one corresponded to the averaged value of each frame (not shown). The second spatial component mostly conveyed movement artifacts, and it could be removed or processed for improving the quality. Components from 3 to about 10 conveyed both spatial variations and spatial artifacts, which are strongly coupled.

\section{Extrapolation in Multimodal Mapping}

To extrapolate the OM signals in the region where the lasso catheter was situated in multimodal mapping, a mask was first manually generated (Fig. 4b). Also, a stage of optical noise canceling by using some of the relevant components from SVD was considered, aiming to study the noise sensitivity of the method. The optical signal was then extrapolated to the regions within the mask. Note that we did not address the image boundaries specifically, as usual in OM preprocessing. Whereas the signal quality in the boundaries seems to be often loose, we wanted to analyze the intrinsic processing for all the sequence, and more, it has not been shown yet that the boundaries will not have relevant information for the global analysis of the OM sequence. The method showed sensitive to the mask segmentation, hence, manual (non automatic) segmentation was made. For extrapolating the frame on the lasso catheter mask region, we used the GPatt as proposed in [4]. This algorithm was chosen because it represents a flexible, non-parametric and computationally tractable approach to 


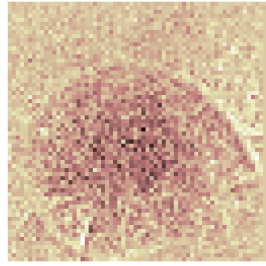

(a)

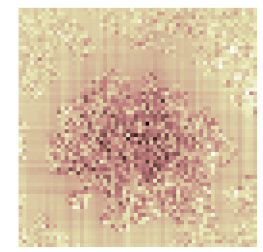

(c)

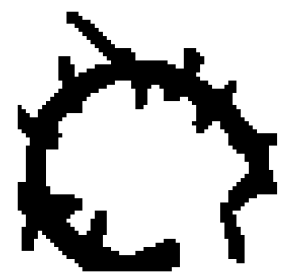

(b)

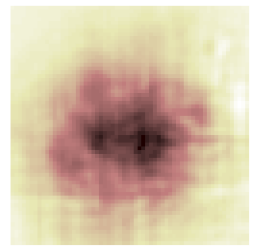

(d)
Figure 4. Frame analysis of GPatt algorithm in multimodal mapping: Original frame and catheter mask $(\mathrm{a}, \mathrm{b})$; extrapolation without and with noise cancelation $(c, d)$.

kernel learning for multidimensional pattern extrapolation, with particular applicability to data with grid structure.

Figure 4 shows the effect of extrapolating without noise canceling, in which the extrapolated signal results in a smoothed version of the mask region, compared to a more similar texture recovered when previously canceling some noise, in this case with the SVD approach. Note that the noise cancellation is not necessarily optimum, but still it allows to scrutinize the interaction between the noise and the texture extrapolation. As an additional validation, we analyzed the extrapolation algorithm in the central known part of the multimodal video, i.e., the square bounded by the lasso catheter. We eliminated a small region in this subimage, which was extrapolated with GPatt. As shown in Fig. 5, the noise is smoothed by the algorithm.

\section{Conclusions}

The use of intrinsic decomposition techniques can provide with enhanced denoising capabilities for processing OM sequences, however, effort has to be devoted on providing with a method for decoupling the spatio-temporal bioelectric activity from the spatio-temporal optical noise artifacts. Other techniques such as Independent Component Analysis or 2-dimensional PCA could provide with enhanced decompositions. Further work has to be devoted to reduce GPatt computational burden, which seems strongly dependent on the noise in the OM sequence.

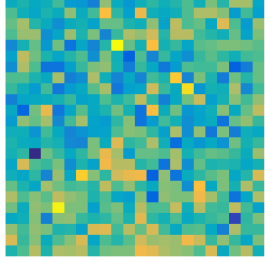

(a)

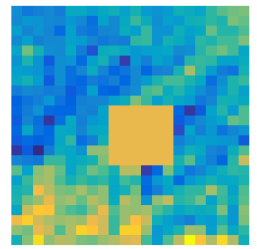

(c)

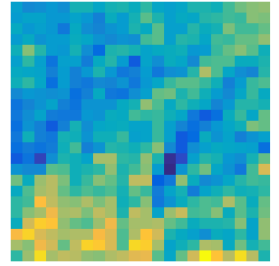

(b)

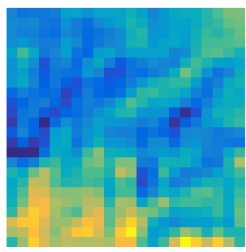

(d)
Figure 5. Validation process for the extrapolation method: (a) Original frame; (b) Frame after SVD; (c) Missing values after SVD; (d) extrapolated image.

\section{Acknowledgements}

This work has been partly supported by Spanish Projects TEC2016-75161-C2-1-4 and TEC2013-48439C4-1-R, and by RD12/0042/0036 (RIC) from Fondo Europeo de Desarrollo Regional and Instituto de Salud Carlos III. CSR is supported by FPU grant AP2012-4225.

\section{References}

[1] Jalife J. And the beat goes on ... the beat goes on: organization and quasi-periodicity in ventricular fibrillation. Cardiovascular Research 2013;99(3):375-377.

[2] Efimov I, Nikolski V, Salama G. Optical imaging of the heart. Circulation Research 2004;95(1):21-33.

[3] Tomii N, Yamazaki M, Arafune T, Honjo H, Shibata N, Sakuma I. Detection algorithm of phase singularity using phase variance analysis for epicardial optical mapping data. IEEE Trans Biomed Eng 2016;63(9):1795-1803.

[4] Wilson A, Gilboa E, Cunningham JP, Nehorai A. Fast kernel learning for multidimensional pattern extrapolation. In Advances in Neural Information Processing Systems 27. Curran Associates, Inc., 2014; 3626-3634.

[5] Golub GH, Van Loan CF. Matrix computations, volume 3. JHU Press, 2012.

Address for correspondence:

Sergio Muñoz-Romero

Area of Signal Theory and Communications

University Rey Juan Carlos. D201, Camino del Molino s/n

28943 - Fuenlabrada (Madrid), Spain

Phone: +34 9148884 62. Mail: sergio.munoz@urjc.es 